\title{
Reelin, Very-Low-Density Lipoprotein Receptor, and Apolipoprotein E Receptor 2 Control Somatic NMDA Receptor Composition during Hippocampal Maturation In Vitro
}

\author{
Mélanie Sinagra, ${ }^{1}$ Danièle Verrier, ${ }^{1}$ Daniela Frankova, ${ }^{2}$ Kimberly M. Korwek, ${ }^{3}$ Jaroslav Blahos, ${ }^{2}$ Edwin J. Weeber, ${ }^{3}$ \\ Olivier J. Manzoni, ${ }^{1}$ and Pascale Chavis ${ }^{1}$ \\ ${ }^{1}$ Institut F. Magendie des Neurosciences, 33077 Bordeaux Cedex, France, ${ }^{2}$ Department of Molecular Pharmacology, Institute of Experimental Medicine, \\ Czech Academy of Science, 14220 Prague 4, Czech Republic, and ${ }^{3}$ Department of Molecular Physiology and Biophysics, Kennedy Center for Research on \\ Human Development, Vanderbilt University Medical Center, Nashville, Tennessee 37232-0615
}

\begin{abstract}
Reelin is a secreted protein that regulates brain layer formation during embryonic development. Reelin binds several receptors, including two members of the low-density lipoprotein (LDL) receptor family, the apolipoprotein E receptor 2 (ApoER2) and the very-low-density lipoprotein receptor (VLDLR). Despite the high level of expression of Reelin and ApoER2 in the postnatal brain, their functions in the adult CNS remain elusive. Here, using electrophysiological, immunocytochemical, and biochemical approaches in cultured postnatal hippocampal neurons, we show that Reelin controls the change in subunit composition of somatic NMDA glutamate receptors (NMDARs) during maturation. We found that maturation is characterized by the gradual decrease of the participation of NR1/2B receptors to whole-cell NMDAR-mediated currents. This maturational change was mirrored by a timely correlated increase of both Reelin immunoreactivity in neuronal somata and the amount of secreted Reelin. Chronic blockade of the function of Reelin with antisense oligonucleotides or the function-blocking antibody CR-50 prevented the decrease of NR1/2B-mediated whole-cell currents. Conversely, exogenously added recombinant Reelin accelerated the maturational changes in NMDA-evoked currents. The maturation-induced change in NMDAR subunits also was blocked by chronic treatment with an inhibitor of the Src kinase signaling pathway or an antagonist of the LDL receptors, but not by inhibitors of another class of Reelin receptor belonging to the integrin family. Consistent with these results, immunocytochemistry revealed that NR1-expressing neurons also expressed ApoER2 and VLDLR. These data reveal a new role for Reelin and LDL receptors and reinforce the idea of a prominent role of extracellular matrix proteins in postnatal maturation.
\end{abstract}

Key words: hippocampus; maturation; Reelin; ApoER2; VLDLR; NMDA receptor

\section{Introduction}

Cell adhesion molecules and components of the extracellular matrix (ECM) are important contributors to both embryonic development and postnatal maturation. In the adult CNS, several forms of synaptic plasticity associated with learning and memory involve ECM-dependent mechanisms reminiscent of those engaged during development (Dityatev and Schachner, 2003). The ECM protein Reelin perfectly illustrates the similarities between

\footnotetext{
Received Nov. 20, 2004; accepted May 16, 2005.

This work was supported by grants from the Institut National de la Santé et de la Recherche Médicale (Programme Avenir), Fondation pour la Recherche Médicale, and Région Aquitaine. We thank Dr. K. Nakajima (Keio University School of Medicine, Tokyo, Japan) for the gift of the CR-50 antibody, Dr. T. E. Willnow (Max Delbrück Center for Molecular Medicine, Berlin, Germany) for the gift of the expression construct of GST-tagged RAP, and C Breillat for the transfection of COS-7 cells. Stably transfected HEK-293 cells expressing recombinant full-length mouse Reelin, originally isolated by G. D'Archangelo and colleagues, were graciously provided by Michael Frotscher and Eckart Förster (University of Freiburg, Freiburg, Germany).

Correspondence should be addressed to P. Chavis, Institut F. Magendie des Neurosciences, 146 rue Leo Saignat, 33077 Bordeaux Cedex, France. E-mail: chavis@bordeaux.inserm.fr.

D0I:10.1523/JNEUROSCI.1757-05.2005

Copyright $\odot 2005$ Society for Neuroscience $\quad$ 0270-6474/05/256127-10\$15.00/0
}

developmental mechanisms and adult synaptic plasticity. Reelin regulates both neuronal migration and positioning during the embryonic development of mammalian brain laminated structures (for review, see D'Arcangelo and Curran, 1998) and plays a role in hippocampal long-term synaptic plasticity (LTP) in the adult brain (Weeber et al., 2002).

Reelin is a large secreted protein (D'Arcangelo et al., 1997) that acts as a signaling molecule. Transmission of the Reelin signal to the target cells requires the binding of Reelin to different classes of receptors, including two members of the low-density lipoprotein (LDL) receptor family: the very-low-density lipoprotein receptor (VLDLR) and the apolipoprotein E receptor 2 (ApoER2) (D'Arcangelo et al., 1999; Hiesberger et al., 1999; Trommsdorff et al., 1999). In addition, Reelin has been reported to bind the integrin $\alpha 3 \beta 1$ (Dulabon et al., 2000) and cadherinrelated neuronal receptors (CNRs) (Senzaki et al., 1999).

In the postnatal hippocampus, Reelin-dependent processes occur via both VLDLR and ApoER2 receptors (Weeber et al., 2002; Niu et al., 2004). Application of either a Reelin-interfering antibody or an antagonist of VLDLR and ApoER2 reduced den- 
dritic outgrowth in cultured postnatal hippocampi (Niu et al., 2004). Application of exogenous Reelin to hippocampal slices produced a significant increase in hippocampal LTP, an effect abolished in slices from mice deficient for VLDLR or ApoER2 (Weeber et al., 2002). Although these studies show the importance of Reelin in postnatal brain functions, the cellular mechanisms underlying LDL receptor-mediated effects on excitatory synaptic transmission and plasticity are not known.

Glutamate receptors of the NMDA subtype (NMDARs) play crucial roles in excitatory synaptic transmission and plasticity. NMDARs are heteromeric ligand-gated ion channels assembled of the obligatory NR1 subunits in combination with NR2 (NR2A-NR2D) or NR3 subunits (Wenthold et al., 2003). The nature of NR2 subunits confers distinct pharmacological and kinetic properties to the heteromeric receptor. In particular, receptors containing NR2A subunits have rapid offset kinetics, whereas receptors containing the other types of NR2 subunits have slower kinetics (Vicini et al., 1998). The expression pattern of the two major NR2 subunits in the hippocampus, NR2A and $\mathrm{NR} 2 \mathrm{~B}$, is regulated developmentally; early in development, NR2B-containing NMDARs are predominant, whereas NR2Acontaining NMDARs prevail at later stages (Monyer et al., 1994; Wenzel et al., 1997). In addition, converging evidence supports the idea that the synaptic NMDA receptor subunit composition can be modified by various factors, such as synaptic activity (Heynen et al., 2000; Barria and Malinow, 2002), experience, or learning (Quinlan et al., 2004) and ECM receptor-dependent maturation (Chavis and Westbrook, 2001).

Here we studied the role of Reelin during postnatal maturation of hippocampal neurons. We report for the first time that Reelin controls changes in the subunit composition of NMDARs during postnatal maturation.

\section{Materials and Methods}

Cell culture and chronic treatments of culture dishes. Hippocampi were dissected from Swiss mice (Janvier, le Genest St. Isle, France) on the day of birth [considered postnatal day $0(\mathrm{P} 0)$ ], subsequently dissociated with the use of papain, and finally triturated mechanically. Cells were plated at a density of 185,000 cells/ml and grown on poly-L-lysine-coated coverslips in MEM supplemented with Serum Supreme (BioWhittaker, Emerainville, France). For chronic treatments, the reagents were added to the culture media $9.5 \mathrm{~d}$ after plating when the ifenprodil-sensitive fraction of whole-cell NMDA currents had reached a plateau (see Fig. $1 \mathrm{~A}$ ). Reagents were replenished at $11 \mathrm{~d}$ in vitro (11 DIV) to avoid possible degradation and then removed by extensive washing before experiments. Concentrations and sources of reagents included the following: $1 \mu \mathrm{M}$ TTX (Alomone Labs, Jerusalem, Israel), $100 \mu \mathrm{M}$ D-AP-5 (Tocris, Ellisville, MO; Fisher Bioblock Scientific, Illkirch, France), $10 \mu \mathrm{M}$ CNQX (Tocris; Fisher Bioblock Scientific), $0.1 \mu \mathrm{m}$ echistatin (Sigma-Aldrich, l'Isle d'Abeau, France), 50-100 $\mu \mathrm{g} / \mathrm{ml}$ hamster anti-mouse $\beta 3$ monoclonal antibody (PharMingen BD Biosciences, Le Pont de Claix, France), 10-30 $\mu \mathrm{M}$ PP1 (Alexis Biochemicals, San Diego, CA; Coger, Paris, France), 10-30 $\mu \mathrm{M}$ PP3 (Calbiochem, La Jolla, CA; VWR International, Strasbourg, France), 30-100 $\mu \mathrm{g} / \mathrm{ml}$ CR-50 antibody (K. Nakajima, Keio University School of Medicine, Tokyo, Japan), 30-100 $\mu \mathrm{g} / \mathrm{ml}$ normal mouse IgG (Jackson ImmunoResearch, West Grove, PA; Interchim, Montluçon, France), 30-100 $\mu \mathrm{g} / \mathrm{ml}$ G10 (Chemicon, Temecula, CA; Euromedex, Mundolsheim, France), $50 \mu \mathrm{g} / \mathrm{ml}$ receptor-associated protein fused with glutathione $S$-transferase (GST-RAP), $50 \mu \mathrm{g} / \mathrm{ml} \mathrm{GST,} 10$ $\mu \mathrm{M}$ Reelin antisense (reelin as), and $10 \mu \mathrm{M}$ control mismatched (reelin $\mathrm{m})$ (Eurogentec, Seraing, Belgium). The sequences of the Reelin phosphorothioate antisense and the corresponding mismatched oligonucleotide were as described previously (Lacor et al., 2000).

Chronic treatments with recombinant Reelin ( $\mathrm{rReelin} ; 1 \mu \mathrm{g} / \mathrm{ml}$ ) or the mock control isolate $(1 \mu \mathrm{g} / \mathrm{ml})$ were performed the day before the date of electrophysiological recordings (i.e., at 6 or 7 DIV to record neurons aged 7 or 8 DIV, respectively).

Electrophysiological recordings and data analysis. Neurons were perfused continuously with $\mathrm{Mg}^{2+}$-free extracellular solution containing the following (in $\mathrm{mM}$ ): $160 \mathrm{NaCl}, 2.4 \mathrm{KCl}, 10 \mathrm{HEPES}, 10$ glucose, 0.02 glycine, and $1.5 \mathrm{CaCl}_{2}, \mathrm{pH} 7.3$, with osmolarity of $325 \mathrm{mOsm} / \mathrm{L}$. The pipette solution contained the following (in $\mathrm{mM}$ ): 149 Cs-methanesulfonate, 1 EGTA, 10 HEPES, $4 \mathrm{MgCl}_{2}$, 10 glucose, and $1 \mathrm{Na}_{2} \mathrm{ATP}, \mathrm{pH}$ 7.3, with osmolarity of 305-310 mOsm/L. To reduce calcium-dependent inactivation (Legendre et al., 1993), we used $0.2 \mathrm{~mm}$ external calcium in agonistand antagonist-containing solutions. Data were acquired with Clampex 8 (Molecular Devices, Union City, CA; DIPSI, Chātillon, France), lowpass-filtered at $2 \mathrm{kHz}$, and digitized at $5 \mathrm{kHz}$. Recordings were discarded if the access resistance was $>5 \mathrm{M} \Omega$ as monitored with $5 \mathrm{mV}$ hyperpolarizing voltage pulses throughout the experiment. Whole-cell NMDA currents were evoked on neurons that were voltage clamped at $-60 \mathrm{mV}$ by $3 \mathrm{~s}$ applications of $1 \mathrm{~mm}$ NMDA (Sigma-Aldrich). Agonist was applied at 30 s intervals until the response was stable. Neurons were equilibrated for $20 \mathrm{~s}$ in $3 \mu \mathrm{M}$ ifenprodil before the application of NMDA plus ifenprodil. Data were analyzed with Clampfit 8 (Molecular Devices; DIPSI). The percentage of ifenprodil block is the charge transfer of the whole-cell NMDA currents evoked in the presence of ifenprodil normalized to the charge transfer of the NMDA-evoked currents before ifenprodil application.

Immunocytochemistry and image analysis. Immunostainings were performed on cells fixed with 4\% PFA, permeabilized with $0.1 \%$ Triton $\mathrm{X}-100$, and subsequently bathed in a blocking buffer. Then the neurons were incubated overnight at $4^{\circ} \mathrm{C}$ (unless stated otherwise) with primary antibodies diluted in blocking solution. After being washed, the cells were incubated with secondary antibodies for $90 \mathrm{~min}$ at room temperature and then washed extensively and mounted on glass coverslips with Aqua-PolyMount (Polysciences, Conches sur Gondoire, France). Reelin was detected with the monoclonal antibody G10 (1:1000; Chemicon; Euromedex) and visualized with a cyanine 3 (Cy3)-conjugated antimouse IgG (Jackson ImmunoResearch; Interchim). The rabbit antiApoER2 (1:200; Santa Cruz Biotechnology, Santa Cruz, CA; Tebu, Le Perray en Yvelines, France) and the monoclonal anti-NR1 (1:250; $2.5 \mathrm{~h}$ at room temperature; PharMingen BD Biosciences) were revealed with a Cy3-conjugated anti-rabbit IgG and a cyanine-5 (Cy5)-conjugated antimouse IgG, respectively. The mouse anti-VLDLR (1:500; Santa Cruz Biotechnology; Tebu) and the rabbit anti-NR1 (1:250; $2.5 \mathrm{~h}$ at room temperature; Chemicon; Euromedex) were visualized with a Cy3conjugated anti-mouse $\operatorname{IgG}$ and a Cy5-conjugated anti-rabbit IgG, respectively.

Slides were viewed with a $40 \times$ oil objective on an Axiophot II microscope (Zeiss, Le Pecq, France). Image acquisitions were done with a Quantix digital cooled CCD camera (Photometrix, Tucson, AZ) driven by IPLab software (Scanalytics, Fairfax, VA). The exposure settings were kept identical for each condition. For each fluorescent image that was acquired, the corresponding phase-contrast field was collected. The images displayed in the figures are overexposed for a better visualization but do not correspond to the images that were analyzed. The 12 bit images were analyzed with MetaMorph Software (Universal Imaging, West Chester, PA; Roper Scientific, Evry, France) as follows. Somata were demarcated on the phase-contrast image, and the regions of interest (ROIs) were transferred automatically on the corresponding fluorescent images. Positive ROIs were selected by intensity threshold calculated as the product of the background by a constant (the estimation of the constant depended on the signal-to-noise ratio in each wavelength). Positive somata then were counted over all of the images acquired on coverslips from one experiment and normalized to the total number of neurons detected on the corresponding phase-contrast images.

Immunoblotting of Reelin in hippocampal lysates. Hippocampi from P0, P7, P14, and P21 Swiss mice were dissected out, immediately homogenized with a hand-held tissue grinder in lysis buffer [ 25 mM HEPES, 150 $\mathrm{mm} \mathrm{NaCl}, 1 \%$ Triton X-100, and protease inhibitor mixture (Roche, Neuilly sur Seine, France)], and subjected to centrifugation $(10,000 \times g$ at $4^{\circ} \mathrm{C}$ for $10 \mathrm{~min}$ ) to remove insoluble material. Protein quantification was performed by using the BCA protein assay kit (Pierce, Rockford, IL; 
Perbio Science, Brebieres, France) according to the manufacturers' instructions. Samples $(40 \mu \mathrm{g}$ of homogenate proteins normalized with tubulin expression) were run through to $6 \%$ SDS-PAGE, using a minigel apparatus (Amersham Biosciences, Orsay, France). The high-molecularweight markers bovine serum albumin and urease (Sigma-Aldrich) were run in parallel to the standard molecular markers ranging from 10 to 250 $\mathrm{kDa}$ (Precision Plus Protein Standards, Bio-Rad, Marnes la Coquette, France). Protein transfer to nitrocellulose membrane was achieved with a liquid blotting apparatus (Amersham Biosciences). Before being immunoblotted, urease and bovine serum albumin were revealed with Ponceau red (Sigma-Aldrich). Reelin was labeled with the monoclonal antibody G10 (1:5000; Chemicon; Euromedex), detected by using an HRPconjugated anti-mouse secondary IgG (1:3000), and visualized with enhanced chemiluminescence (Amersham Biosciences). For each of the three bands of interest, immunoreactivity levels were quantified by integrating band density by area, using computer-assisted densitometry (NIH Image J 1.29). For each gel, the density by area measurements were added over the three bands obtained for the three isoforms at each age (total Reelin). The total protein density obtained for each age (i.e., each lane) was normalized as a percentage of the total protein density at $\mathrm{P} 0 ; n$ represents the number of gels, and each gel was run with samples obtained from different animals.

Western blot analysis of Reelin in cell-culture media and of NR2A/2B/ NR1 and synaptic cell adhesion molecule in cell-culture lysates. Experiments including chronic treatments with Reelin antisense and mismatch as well as the processing of untreated culture dishes during maturation were performed by following a double-blind protocol to avoid any bias.

For immunoblotting of secreted Reelin, the cell media collected from dissociated cultures were concentrated sixfold by using a $50 \mathrm{kDa}$ cutoff filter (Microcon YM-50; Millipore, Molsheim, France). Samples run through to the same $6 \%$ SDS-PAGE were subjected at the same time to protein quantification with the BCA protein assay kit. Immunoblotting and analysis were performed as described above. For each gel, the total protein density obtained for each condition (i.e., each lane) was normalized as a percentage of the total Reelin density at $10 \mathrm{DIV} ; n$ represents the number of gels, and each gel was run with samples obtained from different cultures.

For immunoblotting of NR1, NR2A, and NR2B subunits and synaptic cell adhesion molecule (SynCAM), the neurons were scraped and homogenized in lysis buffer (see above) and subjected to centrifugation $\left(8000 \times g\right.$ at $4^{\circ} \mathrm{C}$ for $15 \mathrm{~min}$ ) to remove insoluble material. Samples (50 $\mu \mathrm{g}$ of homogenate proteins normalized with tubulin expression) were run through to $7.5 \%$ SDS-PAGE, transferred via semi-dry apparatus (Bio-Rad) to nitrocellulose membrane, and probed for the protein of interest. NR1 was probed with a monoclonal antibody (1:1000; PharMingen BD Biosciences). The specificity of rabbit polyclonal antibodies used to label NR2A and NR2B (1:1000; Upstate Biotechnology, Lake Placid, NY; Euromedex) was controlled on African green monkey (COS-7) cells transfected with either NR2A or NR2B (supplemental Fig. 3 , available at www.jneurosci.org as supplemental material). SynCAM was revealed with a rabbit polyclonal antibody (1:3000; Abcam, Cambridge, UK). Labeled proteins were detected with HRP-conjugated antirabbit or anti-mouse secondary antibodies diluted 1:5000 and visualized with enhanced chemiluminescence (Amersham Biosciences). The antibodies against NR1, NR2A, and NR2B recognized a band at $\sim 120,180$, and $170 \mathrm{kDa}$, respectively, and the SynCAM antibody revealed a predominant band $\sim 60-65 \mathrm{kDa}$ (Biederer et al., 2002). Analysis was performed as described above. For each gel, the protein density obtained in each lane was expressed relative to the average protein density at 6 DIV; $n$ represents the number of gels, and each gel was run with samples obtained from different cultures.

Expression of GST fusion construct. Bacterial strain DH5 carrying the GST-RAP expression vector was grown overnight at $37^{\circ} \mathrm{C}$ in $200 \mathrm{ml}$ of LB media (with ampicillin). The culture was mixed with $1800 \mathrm{ml}$ of prewarmed LB/amp and incubated while being shaken vigorously for an additional hour. Isopropyl $\beta$-D-thiogalactoside (Sigma-Aldrich, Prague, Czech Republic) was added to a final concentration of $0.1 \mathrm{~mm}$ for $2 \mathrm{~h}$. Bacteria then were pelleted $(5000 \times g ; 20 \mathrm{~min})$. Pellets were washed twice with $50 \mathrm{ml}$ of buffer $\mathrm{A}(0.1 \mathrm{~mm}$ Tris- $\mathrm{HCl}$ and $0.1 \mathrm{~mm} \mathrm{NaCl}, \mathrm{pH} 7.4)$. After final centrifugation, the pellets were resuspended in $40 \mathrm{ml}$ of buffer A with $4 \mathrm{M}$ urea, and the homogenate was sonicated vigorously. The samples were kept at room temperature for $1 \mathrm{~h}$ and diluted with buffer $\mathrm{A}$ to a final concentration of $100 \mathrm{ml}$. The insoluble was separated by centrifugation $(10,000 \times g$ for $15 \mathrm{~min})$. Supernatants were incubated for $1 \mathrm{~h}$ with $0.5 \mathrm{ml}$ bed volume of glutathione-Sepharose $4 \mathrm{~B}$ (Amersham Biosciences, Uppsala, Sweden) at room temperature. Brief centrifugation was used to concentrate the beads that then were washed in $10 \mathrm{ml}$ of buffer A three times. The beads were resuspended in $1 \mathrm{ml}$ of buffer $\mathrm{A}$ and transferred to $1.5 \mathrm{ml}$ Eppendorf tubes. The protein was eluted with buffer A containing $4 \mathrm{~m}$ urea and $10 \mathrm{~mm}$ glutathione (Sigma-Aldrich, Prague, Czech Republic) in three fractions, $250 \mu \mathrm{l}$ each. Samples were separated on SDSPAGE, and Coomassie blue staining was used for estimation of the protein amount by comparison with protein of a known amount.

Production and purification of recombinant Reelin. Reelin-expressing human embryonic kidney-293 (HEK-293) cells were grown in DMEM high glucose with $10 \%$ fetal bovine serum; untransfected HEK-293 cells were grown in the same manner. Cells then were grown in DMEM low glucose with $0.2 \%$ BSA for $2 \mathrm{~d}$, and the Reelin or mock-conditioned medium was collected, centrifuged, and sterile-filtered. The supernatant was concentrated by using Centricon Plus- 80 centrifugal filter units (Millipore, Bedford, MA). The concentrated medium was purified by size exclusion gel filtration. Fractions that demonstrated immunoreactivity to Reelin (G-20; Santa Cruz Biotechnology) were pooled. Mockconditioned medium was concentrated and purified in the same manner.

Statistical analysis. $n$ is the number of cultures used for each condition. All data are expressed as the mean \pm SEM unless stated otherwise. Statistical analyses were performed by using ANOVA followed, if significant $(p<0.05)$, by Tukey's multiple comparison test (Kyplot $2.0 \beta 13$ ), unless stated otherwise.

\section{Results}

\section{Changes in the functional profile of NR1/NR2B NMDAR- mediated whole-cell currents during maturation}

The NMDAR subunit composition is regulated developmentally (Monyer et al., 1994), and NMDARs of the NR1/2B type can be distinguished from other subtypes of NMDARs on the basis of their high sensitivity toward ifenprodil (Williams, 1993). Thus to evaluate the functional profile of NR1/2B during maturation, we measured the extent of block of whole-cell NMDA-evoked currents by the selective NR1/NR2B receptor antagonist ifenprodil (Fig. $1 A$ ). The percentage of ifenprodil-induced blockade significantly changed between 6 and $12 \operatorname{DIV}\left(F_{(4,22)}=91.25 ; p<\right.$ $0.001)$. The ifenprodil-sensitive fraction of NMDA-evoked currents significantly decreased between 6 and 8 DIV (6 DIV, $55.3 \pm$ $2.1 \% ; 8 \mathrm{DIV}, 47.2 \pm 0.2 \% ; n=5$ and 5 ) and between 8 and 9 DIV $(32.1 \pm 1.3 \% ; n=5)$ and remained stable thereafter (10 DIV, $30.4 \pm 1.2 \%$; 12 DIV, $30.6 \pm 2.4 \% ; n=6$ and 6$)$. This pharmacological sensitivity was supported by a slower inactivation time course of the NMDA-evoked currents at an early stage of maturation, a typical feature of $\mathrm{NR} 1 / 2 \mathrm{~B}$ receptors (Fig. $1 A$, traces) (Vicini et al., 1998). These data indicate that the contribution of NR1/2B receptors to whole-cell NMDA-evoked currents decreased during maturation, a result consistent with the known subunit composition change from NR2B to NR2A (Williams et al., 1993; Monyer et al., 1994).

In some cases, NMDAR subunit expression is known to depend on synaptic activity (Audinat et al., 1994; Heynen et al., 2000; Hoffmann et al., 2000; Barria and Malinow, 2002). To evaluate whether the decline of somatic NR1/2B receptors in 10-12 DIV neurons also was controlled by neuronal activity, we performed chronic treatments (see Materials and Methods) with tetrodotoxin (TTX) to block action potentials or a mixture of the NMDAR antagonist D-AP-5 and the non-NMDAR antagonist CNQX to block excitatory synaptic transmission. Chronic arrest of neuronal activity did not significantly change the ifenprodil 
sensitivity of whole-cell NMDA-evoked currents in 10-12 DIV neurons when compared with untreated neurons at the same age (Fig. $1 B$ ). Thus the changes in the NMDAR subunit composition that occur between 9 and 12 DIV do not depend on neuronal activity. We next searched for the mechanisms underlying the maturational changes in NMDAR subunit composition and properties in hippocampal neurons in vitro.

\section{Activity-independent maturation of NMDAR currents is independent of integrins}

We have demonstrated previously the role of the cell adhesion family of integrins in the activity-dependent maturation of hippocampal synapses occurring at earlier stages in vitro (4.5-8 DIV) (Chavis and Westbrook, 2001); thus we tested the possibility of a role of this family of adhesion molecules in the maturation of NMDARs between 9 and 12 DIV. Chronic treatment with echistatin, a snake toxin that preferentially inhibits the binding of ECM ligands to $\beta 1$ - or $\beta 3$-containing integrins (Thibault, 2000), did not prevent the maturation-induced reduction in ifenprodil sensitivity of wholecell NMDA-evoked currents (Fig. $1 B$ ). Furthermore, chronic treatment with a function-blocking antibody directed against the integrin subunit $\beta 3$ (Chavis and Westbrook, 2001) had no effect on the extent of ifenprodil inhibition of whole-cell NMDAevoked currents (Fig. $1 B$ ). Together, these data showed that $\beta 1$ or $\beta 3$-containing integrins do not participate in maturationinduced reduction of NR2B subunits in 9-12 DIV neurons. Thus the mechanisms involved in the early activity-dependent phases of maturation do differ markedly from the mechanism underlying the maturation-induced changes in NMDAR subunits in 9-12 DIV neurons.

\section{Activity-independent maturation of NMDAR currents involves the Src family tyrosine kinase}

Based on the fact that the Src family of nonreceptor tyrosine kinases regulates NMDAR-dependent plasticity (for review, see Ali and Salter, 2001) and that plasticity and maturation share common mechanisms (for review, see Dityatev and Schachner, 2003), we decided to perform a chronic treatment with PP1, an inhibitor of the Src family tyrosine kinases (Hanke et al., 1996).

In marked contrast to what was observed with integrin blockers, chronic treatment with PP1 significantly altered the ifenprodil sensitivity of whole-cell NMDA-evoked currents $\left(F_{(4,19)}=\right.$ 53.16; $p<0.001$ ) (Fig. $1 B$ ). The extent of ifenprodil blockade increased greatly when compared with age-matched neurons and was indistinguishable from that of 8 DIV neurons (Fig. 1, compare $A, B)$. The structurally related inactive compound $\mathrm{PP} 3$, used as a control, was without effect. To test whether the acute regulation of NMDA channel activity by the Src family kinases (Yu et al., 1997) was responsible for the maturational switch in NMDA subunits, we performed short-term treatments (1-6 h; $30 \mu \mathrm{M}$ ). Such treatments did not modify the ifenprodil sensitivity of NMDA-evoked currents when compared with untreated conditions (Fig. $1 B$ ). ces of whole-cell NMDA currents in the absence (black traces) or in the presence (gray traces) of ifenprodil ( $3 \mu \mathrm{m})$ in $6-12$ DIV Chronic treatment with PP1 increased the ifenprodil sensitivity of NMDA currents to $48.3 \pm 0.9 \%(n=5)$. Neither PP3 nor acute

B

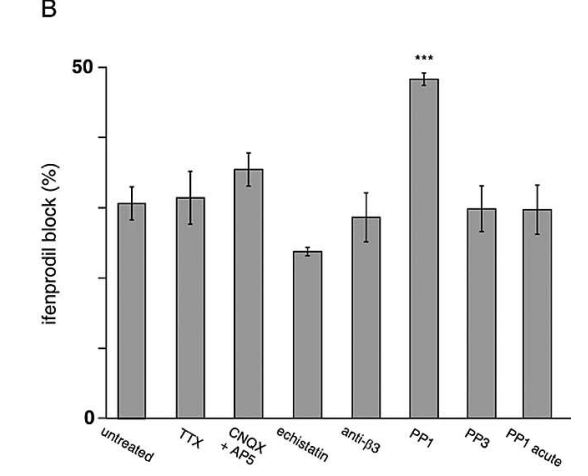
10 DIV 12 DIV

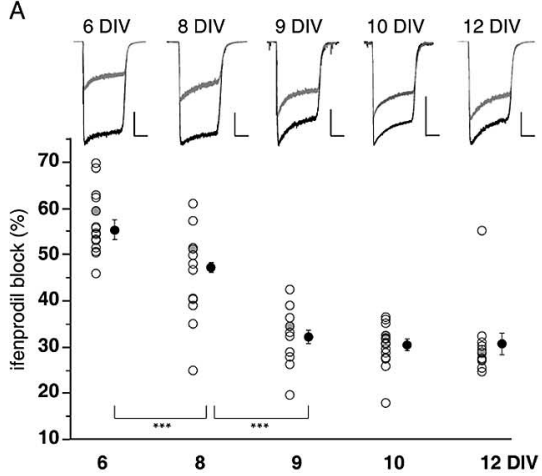

o 
A

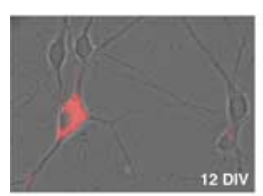

B

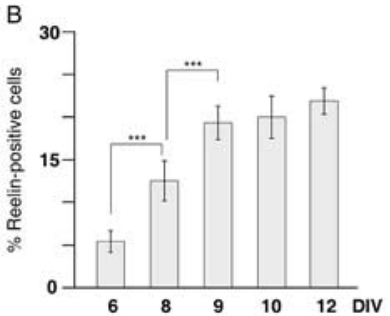

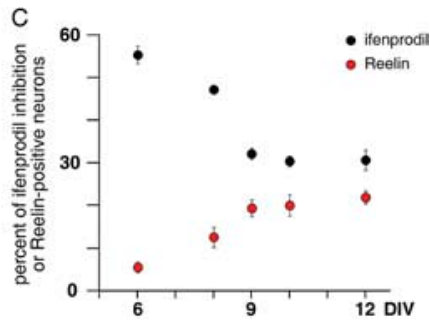

Figure 2. Correlated changes in Reelin expression and ifenprodil sensitivity of NMDA currents during maturation. $\boldsymbol{A}$, Representative image taken from a 12 DIV culture showing the characteristic strong somatic labeling found in Reelin-immunoreactive neurons (red). The pseudocolored image is superimposed on the corresponding phase-contrast image. $\boldsymbol{B}$, Changes in the proportion of Reelin-positive somata during maturation. The number of Reelin-immunoreactive somata is expressed as the percentage \pm SEM of the total number of cell bodies counted on the corresponding phase-contrast image. The percentages of Reelinpositive cells were $5.4 \pm 1.3 \%$ at 6 DIV $(n=4), 12.6 \pm 2.4 \%$ at 8 DIV $(n=5), 19.4 \pm 2.0 \%$ at 9 DIV $(n=5), 20.0 \pm 2.5 \%$ at 10 DIV $(n=3)$, and $21.9 \pm 1.6 \%$ at 12 DIV $(n=3) .{ }^{* *} p<0.001$. C, Comparison of the time course of the ifenprodil blockade of NMDA-evoked currents (black circles) and of the Reelin immunoreactivity (red circles) between 6 and 12 DIV.
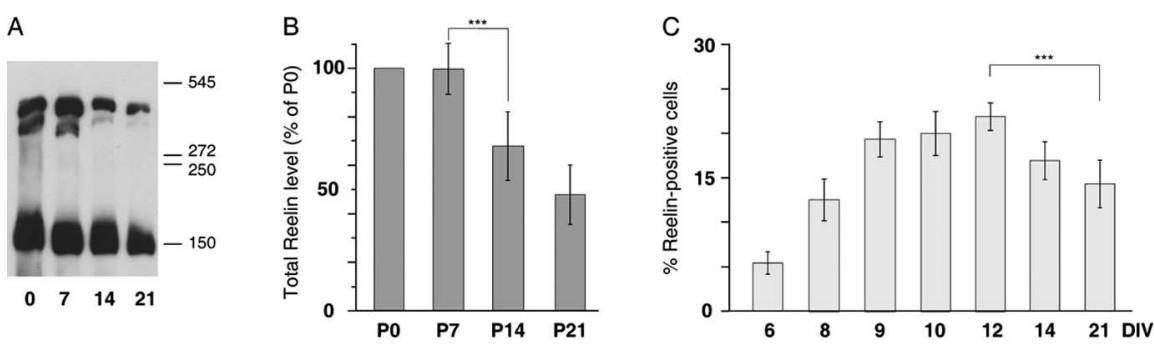

Figure 3. Comparison of the hippocampal maturation profile of Reelin in vivo and in vitro. $\boldsymbol{A}$, Representative Western blot of Reelin in hippocampi from Swiss mice at different postnatal ages (P0-P21). Three isoforms of Reelin were detected with the use of the G10 antibody (D'Arcangelo et al., 1999; Lambert de Rouvroit et al., 1999; Lacor et al., 2000). We calculated their respective molecular weights on the basis of their electrophoretic mobility. The molecular weights of the standard proteins, bovine serum albumin, and urease were plotted against the mobility of each protein. The molecular weights of the three Reelin isoforms were estimated to be $406 \pm 21,359 \pm 19$, and $179 \pm 9 \mathrm{kDa}(n=5)$. These estimated molecular weights corresponded to the full-length protein of $\sim 400 \mathrm{kDa}$ and two proteolytic products of $\sim 320$ and $180 \mathrm{kDa}$, as described previously (Lacor et al., 2000). $\boldsymbol{B}$, Densitometry measurements of total Reelin (sum of the 3 isoform densities) at each age are expressed as the percentage \pm SD of P0 $(n=6)$. Significant changes in the protein levels are observed between $\mathrm{P} 0$ and $\mathrm{P} 21\left(F_{(2,15)}=12.59\right.$; $\left.^{* * *} p<0.001\right)$. The protein levels significantly decreased between $\mathrm{P} 7(99.7 \pm 10.4)$ and P14 (68.0 \pm 5.8$)$ and remained stable between P14 and P21 $(47.9 \pm 5.0)$. C, Proportions of Reelin-positive somata between 6 and 21 DIV (same as in the legend to Fig. $2 B$ ). Statistical analysis showed significant differences $\left(F_{(6,23)}=5.03 ;{ }^{* * *} p<0.01\right)$. The percentage of Reelin-immunoreactive neurons was $16.94 \pm$ $2.13(n=4)$ at 14 DIV and $14.33 \pm 2.97(n=6)$ at 21 DIV.

observed between 6 and 9 DIV was correlated tightly with an increase in Reelin immunoreactivity during the same period. Between 9 and 12 DIV, both markers reached a steady-state level (Fig. 2C). In light of these results, we tested the putative role of Reelin in the maturational changes affecting NMDA-mediated currents. We decided to start chronic treatments of the cultures at 9.5 DIV, a time at which both maturational markers (Reelin immunoreactivity and functional NR1/2B receptors) were stabilized.

\section{Blocking the expression or function of Reelin prevents maturation}

First we observed that chronic blockade of Reelin biosynthesis with an oligonucleotide antisense, reelin as (Lacor et al., 2000), mimicked the effect of Src family kinase blockade and significantly increased the ifenprodil sensitivity of whole-cell NMDAevoked currents $\left(F_{(3,17)}=73.55 ; p<0.001\right)$ (Fig. $\left.4 A\right)$. Indeed, when Reelin biosynthesis decreased starting from 9.5 DIV, the extent of block of whole-cell NMDA currents by ifenprodil in 10-12 DIV neurons recovered to what was measured in 8 DIV neurons (reelin as, $47.8 \pm 1.9 \%, n=5 ; 8 \mathrm{DIV}, 47.2 \pm 0.2 \%, n=$ 5) (compare Figs. $1 \mathrm{~A}, 4 \mathrm{~A}$ ). The mismatched antisense reelin $\mathrm{m}$

did not perturb maturation (reelin $\mathrm{m}$, $30.1 \pm 2.5 \%, n=5$; age-matched untreated, $30.6 \pm 2.4 \%, n=6$ ) (Fig. $4 A$ ).

To address further the role of Reelin in the maturational pattern of $\mathrm{NR} 1 / 2 \mathrm{~B}$ NMDAR, we examined the effect of a chronic treatment with the monoclonal antibody CR-50, which blocks the function of Reelin both in vivo (Nakajima et al., 1997) and in vitro (Miyata et al., 1997). Chronic blockade of the function of Reelin reverted the mature phenotype of 10-12 DIV cells $\left(F_{(4,15)}=39.68 ; p<0.001\right)$ (Fig. $4 A)$. Compared with age-matched neurons treated with the G10 antibody or a normal mouse IgG (used as controls), the ifenprodil-sensitive fraction of whole-cell evoked NMDA currents in 10-12 DIV neurons increased greatly after chronic treatment with the CR-50 antibody (CR$50,47.1 \pm 1.9 \%, n=4 ; \mathrm{G} 10,30.3 \pm 0.6 \%$, $n=3$; IgG, $31.1 \pm 0.7 \%, n=3$ ) (Fig. $4 A$ ). The ifenprodil-induced inhibition in 10-12 DIV neurons chronically treated with CR-50 was indistinguishable from the extent of block observed both in reelin astreated neurons and in 8 DIV neurons (Fig. $1 A$ ). These results demonstrate that interfering with the synthesis of Reelin (with reelin as) or its ability to bind to its putative receptors (with the CR-50 antibody) prevents the diminution of functional NR1/2B receptors in 10-12 DIV neurons, and the results provide strong evidence that Reelin is a key element of the late, activity-independent maturation of NMDAR currents.

To examine whether changes in the total cellular amounts of NR subunit protein could underlie the functional changes observed during maturation and after chronic treatment with the Reelin antisense, we performed Western blot analysis of the NR2A, NR2B, and NR1 subunits (Fig. 4B). No significant changes were observed in total NR2A and NR2B protein expression either between 6 and 10 DIV $(p>0.05$; paired $t$ test) or after chronic treatment with reelin as or reelin $\mathrm{m}(p>$ 0.05 ; paired $t$ test). On the contrary, the profile of the NR1 subunit protein displayed a small but significant increase between 6 and 10 DIV (6 DIV, $100.0 \pm 14.6 \%$; 10 DIV, $145.0 \pm 21.9 \%$; $n=$ 6 ; $p<0.05$; paired $t$ test). The expression level of NR1 was not altered by chronic treatment with the antisenses (reelin as, $141.8 \pm 31.6 \%$; reelin $\mathrm{m}, 128.2 \pm 22.0 ; n=6 ; p>0.05$; paired $t$ test). Furthermore, we made sure that chronic blockade of Reelin function did not perturb another maturational process. We verified that the expression of the cell adhesion molecule SynCAM changed during maturation in our in vitro assay (supplemental Fig. 1, available at www.jneurosci.org as supplemental material), consistent with the developmental profile described in vivo (Biederer et al., 2002). The maturation-regulated expression of SynCAM was not altered by chronic treatment with the Reelin antisense (supplemental Fig. 1, available at www.jneurosci.org as supplemental material). 
A

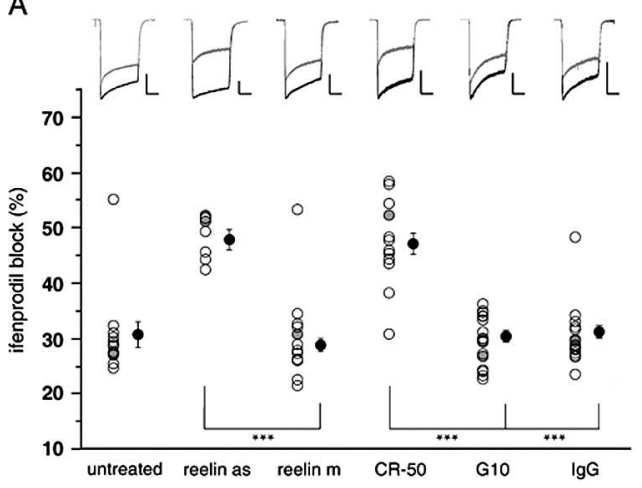

B
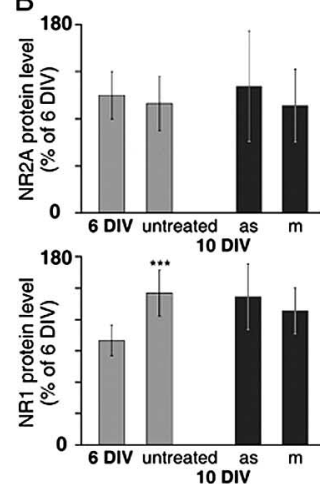

C

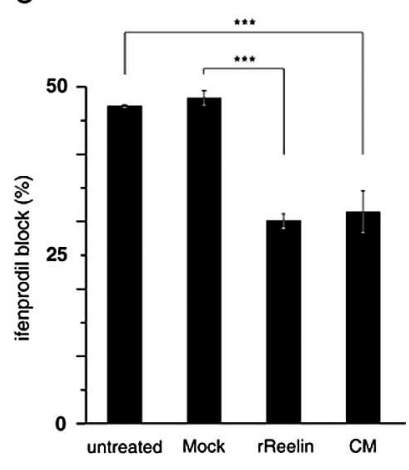

Figure 4. Reelin controls maturational changes in somatic NMDAR subunit composition. $A$, Effect of chronic blockade of the biosynthesis or function of Reelin on ifenprodil-induced inhibition of NMDA currents. A scatterplot of the percentage of ifenprodil block on NMDA-evoked currents recorded in $10-12$ DIV neurons when untreated, after a chronic treatment with the antisense reelin as or the control mismatched reelin $\mathrm{m}$, with the function-blocking antibody CR-50, with the G10 monoclonal antibody as a control, or with a control mouse lgG is shown. For each condition, representative traces of whole-cell NMDA-evoked currents are shown in the absence (black traces) or in the presence (gray traces) of ifenprodil. Calibration: $500 \mathrm{pA}, 1 \mathrm{~s}$. In each group, the gray circles correspond to the percentage of ifenprodil-induced inhibition obtained for the top traces: 26.9,51.2, 30.7,52.1,27.8, and 30.4, respectively. Black circles represent the ensemble average \pm SEM of the percentage of ifenprodil-induced inhibition obtained in each group. See Materials and Methods for chronic treatments. ${ }^{* * *} p<0.001 . B$, Densitometric measures of total NR2A, NR2B, and NR1 protein levels from Western blots of hippocampal cell cultures taken at various ages during maturation either in untreated conditions $(6,8,9,10$, and 12 DIV) or after chronic treatment with the Reelin antisense (as) or the control mismatched $(\mathrm{m})$. Results are expressed relative to the mean of densitometry values obtained at 6 DIV \pm SEM $(n=5)$. Total cellular NR2A protein levels remained relatively constant in all conditions ( 6 DIV, $100.0 \pm 20.2 \% ; 10$ DIV, $93.0 \pm 23.1 \%$; 10 DIV cultures chronically treated with either as or $\mathrm{m}, 107.6 \pm 46.9$ and $91.3 \pm 31.1 \%$, respectively; $n=5$ ) as did NR2B protein levels (6 DIV, $99.9 \pm 17.3 \%$; 10 DIV, $99.7 \pm 11.6 \% ; 10$ DIV as, $84.7 \pm 18.5 \% ; 10$ DIV m, $75.6 \pm 14.4 \% ; n=4) .{ }^{* * *} p<0.05$. C, Effect of exogenously added recombinant Reelin on the ifenprodil sensitivity of whole-cell NMDA-evoked currents recorded in 8 DIV neurons. The average \pm SEM of ifenprodil-induced inhibition of NMDA currents in 8 DIV neurons was $47.2 \pm 0.2 \%$ $(n=5)$ in the untreated condition and $48.4 \pm 1.1 \%(n=4)$ in mock-treated neurons. Treatment with rReelin or CM decreased the ifenprodil sensitivity of NMDA currents to $30.1 \pm 1.1 \%(n=$ 4) and $31.5 \pm 3.1 \%(n=3)$, respectively. ${ }^{* *} p<0.001$.

A

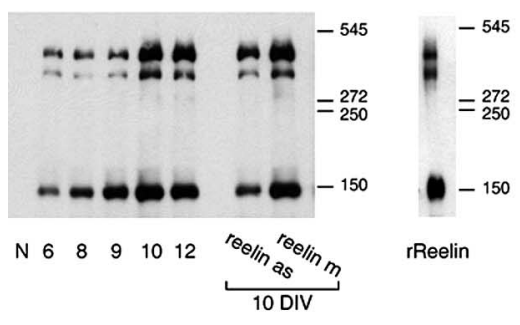

B

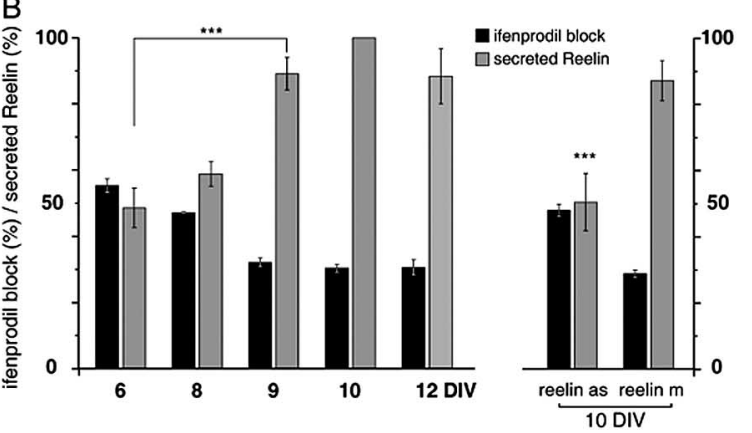

C

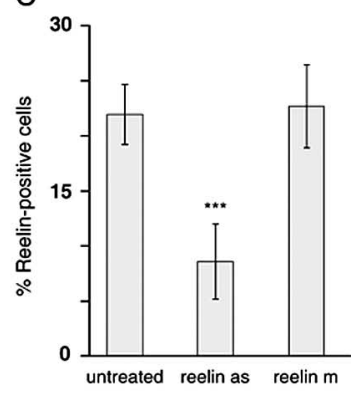

Figure 5. Profile of secreted Reelin during in vitro maturation and effect of antisense oligonucleotides against Reelin. $A$, Representative Western blot of Reelin secreted in the medium of hippocampal cell cultures during maturation in untreated conditions $(6,8,9,10$, and 12 DIV) and in 10 DIV cultures chronically treated with reelin as or the control reelin $\mathrm{m}$. Bands corresponding to full-length Reelin and proteolytic products were detected. Medium not exposed to cultures was included as a negative control (N), and rReelin was included as a positive control. $\boldsymbol{B}$, Coordinated changes in Reelin secretion (gray bars) and ifenprodil-induced blockade of NMDA-evoked currents (black bars) either during maturation in untreated cultures or in 10 DIV cultures chronically treated with reelin as or reelin $\mathrm{m}$. Densitometry measurements of total Reelin (sum of the densities of the 3 isoforms) in each condition are expressed as the percentage \pm SEM of 10 DIV untreated ( $n=5$ ). ${ }^{* * *} p<0.001$. C, Effect of chronic blockade of the biosynthesis of Reelin on Reelin immunoreactivity. The number of Reelin-immunoreactive somata expressed as the percentage \pm SD of the total neuron number counted on the phase-contrast image in $10-12$ DIV cultures either untreated or chronically treated with the antisense oligonucleotides against Reelin (reelin as, $8.5 \pm 3.4 \%$; $n=$ 4) or the mismatched antisense (reelin $\mathrm{m}, 22.6 \pm 3.8 \% ; n=4$ ) is shown. ${ }^{* * *} p<0.01$.

Recombinant Reelin accelerates the maturation-induced changes in functional NR1/2B NMDAR-mediated currents To evaluate whether Reelin is necessary and sufficient to induce maturation, we examined the effect of exogenously added rReelin on the extent of ifenprodil-induced blockade of whole-cell NMDA currents in 8 DIV neurons. Chronic treatment with rReelin (see Materials and Methods) altered the ifenprodil sensitivity of NMDA currents in these neurons $\left(F_{(3,15)}=82.94 ; p<0.001\right)$ (Fig. 4C). Compared with age-matched neurons, the extent of the ifenprodil blockade decreased greatly in rReelin-treated neurons. In fact, the ifenprodil-induced inhibition of NMDA-evoked currents was indistinguishable from that of 10-12 DIV neurons (compare with Fig. 1A). A similar effect was obtained in 7 DIV neurons (untreated, $51.1 \pm 8.1 \%, n=3$; rReelin, $30.9 \pm 0.9, n=$ 3) (results not shown). As a control, the mock control isolate was without effect ( $48.4 \pm 1.1 \% ; n=4$ in 8 DIV neurons) (Fig. $4 C$ ) (51.0 $\pm 2.8 ; n=3$ in 7 DIV neurons) (data not shown). Given that rReelin is composed of a mixture of three isoforms of 180, 320 , and $400 \mathrm{kDa}$ (Fig. 5A), the amount of rReelin used in chronic treatments corresponds to a concentration of $2.50-5.55 \mathrm{~nm}$. This value is similar to the concentration that is efficient at enhancing LTP in area CA1 brain (Weeber et al., 2002) and to the concentration of Reelin that produced a maximal response on dendrite outgrowth (Niu et al., 2004). 
A

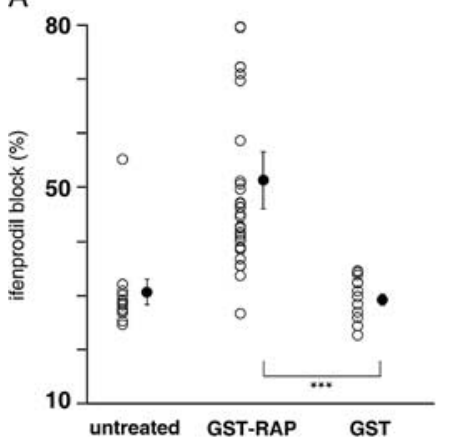

B

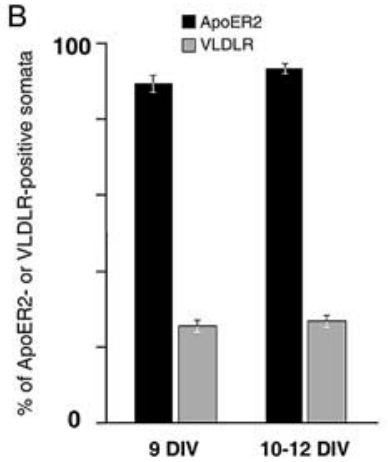

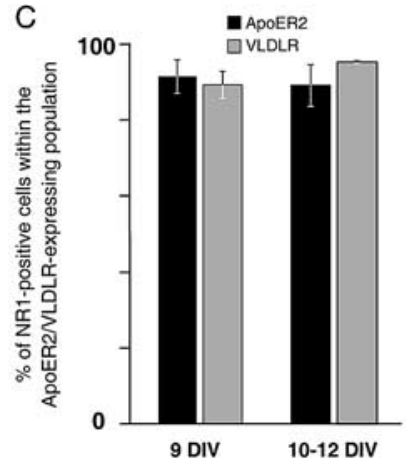

D

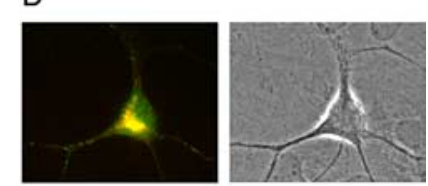

$\mathrm{E}$

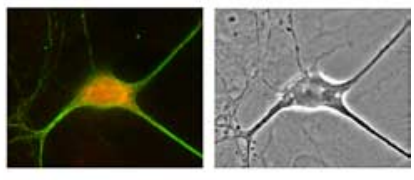

Figure 6. Role and expression profile of the Reelin receptors ApoER2 and VLDLR between 9 and 12 DIV. $A$, Effect of chronic treatment with GST-RAP or GST alone on the ifenprodil-induced inhibition of NMDA-evoked currents in 10-12 DIV neurons (same legend as in Figs. $1 A, 4 A)$. The ifenprodil-sensitive fraction was $51.3 \pm 8.9(n=5)$ in GST-RAP-treated neurons and $29.3 \pm 0.6$ $(n=5)$ after a chronic treatment with GST. ${ }^{* * *} p<0.001$. B, ApoER2 and VLDLR immunoreactivities in 9 and $10-12$ DIV neurons. The histogram shows the proportion of ApoER2-expressing (black bars) or VLDLR-expressing (gray bars) somata expressed as the percentage \pm SEM of the total number of cell bodies counted on the corresponding phase-contrast image. The proportion of ApoER2-positive neurons was $89.2 \pm 2.2 \%(n=5)$ at 9 DIV and $93.5 \pm 1.3 \%(n=4)$ between 10 and 12 DIV $(p>0.05)$. At 9 DIV, $25.5 \pm 1.4 \%(n=4)$ of the total neuronal population was VLDLR-immunoreactive, a percentage not different between 10 and 12 DIV $(26.6 \pm 1.6 \% ; n=6 ; p>0.05)$. C, Quantitative analysis of double labeling of NR1/VLDLR or NR1/ApoER2. The histogram shows the percentage \pm SEM of NR1-positive somata detected within the population of ApoER2-immunoreactive neurons [9 DIV, $91.5 \pm 4.5 \%(n=4) ; 10-12 \mathrm{DIV}, 89.1 \pm 5.6 \%(n=3) ; p>0.05$ ] or VLDLR-expressing neurons [9 DIV, $89.2 \pm 3.4 \%(n=4) ; 10-12$ DIV, $95.6 \pm 0.2 \%(n=3) ; p>0.05$ ]. D, E, Pseudocolored images showing representative double labeling of a VLDLR-positive (red) and an NR1-positive (green) neuron (D) and of an ApoER2-containing (red) and an NR1-containing (green) neuron (E). The corresponding phase-contrast images are shown on the right.

We also controlled so that rReelin did not interact directly with NMDAR by acutely treating hippocampal neurons with rReelin (same concentration as above) and measuring NMDA currents evoked in the presence of rReelin. Moreover, to test whether acute Reelin could affect NMDARs depending on their subunit composition, we performed experiments on both 6 and 10 DIV neurons. As shown in supplemental Figure 2 (available at www.jneurosci.org as supplemental material), perfusion of hippocampal neurons for up to $7 \mathrm{~min}$ with rReelin did not alter NMDA currents, showing that Reelin does not interact directly with NMDARs.

In light of these results, the effect of Reelin endogenously secreted in the medium of hippocampal cultures was also tested. Neurons were treated chronically with conditioned medium (CM) collected from 14 DIV cultures. This treatment closely mimicked the effect of rReelin. The ifenprodil sensitivity of whole-cell NMDA-evoked currents recorded in 8 DIV neurons decreased significantly when compared with age-matched untreated neurons and reached a level indistinguishable from that of $10-12$ DIV neurons $\left(F_{(3,14)}=34.08 ; p<0.001\right)$ (compare Figs. $1 A, 4 C)$.

\section{Changes in the Reelin content of the culture medium during maturation}

We quantified the levels of secreted Reelin into the medium of hippocampal cultures during maturation (Fig. $5 A, B$ ). The content of secreted Reelin significantly changed between 6 and 12 $\operatorname{DIV}\left(F_{(3,25)}=13.19 ; p<0.001\right)$. The total levels of Reelin in culture media significantly increased between 6 and 9 DIV (6 DIV, $48.6 \pm 6.0 \%$; 8 DIV , $58.8 \pm 3.7 \%$; 9 DIV , $89.1 \pm 4.9 \%$; $n=$ 5 ) and remained stable between 9 and 12 DIV (12 DIV, $88.3 \pm$ $8.3 ; n=5$ ). As a control, it was verified that Reelin was not detected in medium not exposed to cultured neurons (Fig. $5 A$, lane N). This pattern was mirrored by the profile of Reelinimmunoreactive neurons (Fig. 2 B) and was correlated in a timely manner with the changes we observed in the functional profile of NR1/2B receptors during the same period (Fig. 5B, black bars). As expected, chronic treatment with the oligonucleotide reelin as significantly decreased the total levels of secreted Reelin when compared with age-matched cultures treated with the control mismatched (Fig. $5 B$, gray bars). The total level of Reelin in reelin as-treated culture dishes was indistinguishable from the level present in 6 or 8 DIV culture media $\left(F_{(5,35)}=15.12 ; p<0.001\right)$. The mismatched antisense was without effect when compared with untreated 10 and 12 DIV cultures (Fig. 5B). Again in these experimental conditions, the level of Reelin immunoreactivity was correlated tightly with the proportion of functional NR1/2B NMDARs (Fig. 5B).

The reelin as-induced decrease in the content of secreted Reelin was mirrored by a decrease in Reelin-positive cells (Fig. 5C). Indeed, chronic treatment with the oligonucleotide reelin as reduced the number of Reelin-positive somata to a level normally observed in 6 or 8 DIV cultures $\left(F_{(2,8)}=12.96\right.$; $\left.p<0.01\right)$ (compare Figs. $2 B, 5 C)$. It was verified that the number of Reelinpositive cell bodies was not changed after chronic treatment with the mismatched oligonucleotide when compared with agematched untreated (Fig. 5C).

\section{Role of ApoER2 and VLDLR receptors in the maturation of NMDAR-mediated currents}

Reelin binds to different classes of receptors, including VLDLR and ApoER2 (D’Arcangelo et al., 1999; Hiesberger et al., 1999). It is noteworthy that the CR-50 function-blocking antibody has been shown previously to "block the association of Reelin with VLDLR” (D’Arcangelo et al., 1999). To determine whether LDL receptors mediate the effects of Reelin in the maturation of NMDAR subunits, we chronically treated our cultures with RAP fused with GST. GST-RAP is a well described tool that prevents the binding of all known ligands to members of the LDL receptor family (Herz et al., 1991), including the binding of Reelin to both VLDLR and ApoER2 (Hiesberger et al., 1999). Moreover, GSTRAP has been used mainly to disrupt physiological functions of Reelin (Hiesberger et al., 1999; Weeber et al., 2002; Niu et al., 2004). Chronic treatment of our cultured neurons with GSTRAP clearly mimicked the effect of neutralizing the function of Reelin (with reelin as and CR-50 treatments) and increased the ifenprodil sensitivity of whole-cell-evoked NMDA currents $\left(F_{(3,16)}=10.60 ; p<0.001\right)$ (Fig. $\left.6 A\right)$. A similar concentration of 
control GST had no effect on the ifenprodil sensitivity of NMDA currents when compared with untreated age-matched cultures.

We then analyzed the expression pattern of VLDLR and ApoER2 by immunocytochemistry in 9 and 10-12 DIV neurons (Fig. 6B-E). In agreement with a previous report (Niu et al., 2004), ApoER2 was expressed in most hippocampal neurons, whereas VLDLR-positive neurons represented a much smaller population. We found that the proportion of neurons expressing VLDLR or ApoER2 did not significantly vary during this period (Fig. $6 \mathrm{~B}$ ). Because GST-RAP prevented the maturation-induced change in NMDAR subunit composition, we decided to perform double labeling of ApoER2 or VLDLR with NR1 (Fig. 6C-E). Quantitative analysis showed that the vast majority of ApoER2or VLDLR-expressing neurons were also NR1 positive (Fig. 6C). This result is consistent with the fact that chronic treatment with GST-RAP impaired the changes in ifenprodil sensitivity of NMDA currents in almost all of the neurons that were recorded (Fig. 6A). The proportion of double-labeled NR1-positive and ApoER2/VLDLR-positive neurons was stable between 9 and 12 DIV (Fig. 6C).

Together, these results show the involvement of members of the LDL receptor family in postnatal maturation-dependent changes in NMDAR subunits and also confirm the instrumental role of Reelin in this process.

\section{Discussion}

In the CNS, various ECM components, which are expressed strongly during development, also remain present during brain maturation and adulthood. Several ECM proteins and their adhesion receptors are involved in different types of remodeling processes occurring in the adult and postnatal brain, such as normal synaptic plasticity (Chun et al., 2001) or maturation (Chavis and Westbrook, 2001). The main finding of this study is that Reelin, an ECM component already known to be recruited in long-term plasticity in the adult hippocampus (Weeber et al., 2002), also is involved in postnatal hippocampal maturation.

The relative contribution of NR2A and NR2B subunits to native NMDARs varies during development. The in vivo development of the hippocampus is characterized by a switch from a predominance of NMDARs rich in NR2B subunits to NMDARs rich in NR2A subunits (Monyer et al., 1994; Wenzel et al., 1997). Consistent with this developmental profile, we showed that the proportion of functional $\mathrm{NR} 1 / 2 \mathrm{~B}$ receptors participating in whole-cell evoked NMDA currents gradually decreased during the maturation of postnatal hippocampal neurons in our in vitro preparation of dissociated neurons. Thus we took advantage of these changes in the subunit composition of NMDARs observed between 6 and 12 DIV and used the ifenprodil sensitivity of whole-cell evoked NMDA currents as a functional index of maturation.

We found that the pattern of ifenprodil sensitivity was mirrored by the expression profile of Reelin. The plateau of Reelin expression was reached between 9 and 12 DIV and accounted for $\sim 20 \%$ of the cultured neurons, a value similar to and comparable with the peak values reported by Scotti and Herrmann (2002) in hippocampal cultures. This plateau was preceded by a gradual increase in the percentage of Reelin-positive neurons between 6 and 9 DIV and was followed by a decline between 14 and 21 DIV. This bell-shaped pattern is similar to what has been described in the embryonic and early postnatal hippocampus in vivo by Alcantara et al. (1998). We also followed the modifications of Reelin by Western blot from whole hippocampi taken at birth (P0) and early postnatal ages (P7, P14, and P21). We found a steady-state level of total Reelin expression in P0 and P7 hippocampi and a decline between P7 and P14. This pattern closely matches the mRNA distribution profile observed by Alcantara et al. (1998) in postnatal hippocampus. Thus we provide evidence not only that Reelin expression is maintained in dissociated cultures of neonatal hippocampal neurons but also that its expression profile is similar, albeit delayed, to the pattern observed in vivo by immunoblots in early postnatal hippocampi (taken from the same strain of mice) and by in situ hybridization analyses in the developing and postnatal hippocampus (from a different strain of mice) (Alcantara et al., 1998).

A central observation of the present study is that impairment of Reelin biosynthesis with the previously described Reelin antisense (Lacor et al., 2000) and inhibition of Reelin activity with the interfering antibody CR-50 prevented the decrease in ifenprodilsensitive NMDA-evoked currents that normally occurs during maturation. The antisense approach allowed us to block Reelin function at a chosen time in neurons that had started their maturation in a nonperturbed environment. Conversely, we report for the first time that exogenous recombinant Reelin or conditioned medium collected from older cultured neurons can accelerate the maturational-induced changes in functional NMDAR subunits.

The changes in the functional profile of NR1/2B NMDARmediated whole-cell currents during maturation were accompanied by a stable cellular expression of NR2A and NR2B and by a small but significant increase in the total NR1 protein level. Thus our findings are not consistent with changes at the synthesis level as the principal mode for regulating the functional changes in whole-cell NMDA currents during maturation. Because NR2A and NR2B cellular expression remained unaffected by chronic treatment with the antisense against Reelin, this suggests that the effect of Reelin blockade on NR1/2B-mediated whole-cell currents involves more complex mechanisms, such as regulation of trafficking, changes in the surface delivery of receptors, or alteration of the lateral mobility of NMDARs (Triller and Choquet, 2005)

The Reelin signal transduction involves binding to different families of receptors, such as ApoER2 or VLDLR (D'Arcangelo et al., 1999), the integrin $\alpha 3 \beta 1$ (Dulabon et al., 2000), or the protocadherins CNR1-CNR8 (Senzaki et al., 1999), although the interaction between Reelin and CNRs has been questioned recently (Jossin et al., 2004). The effect of RAP, a general inhibitor of ligand binding to LDL receptor family members, is in agreement with the involvement of the receptors ApoER2 and/or VLDLR in the Reelin-dependent phase of maturation. RAP also blocks other neuronally expressed receptors, such as LDL receptor-related protein 1 and LDL receptor (Beffert et al., 2004), but these receptors have never been described as binding Reelin. The fact that the disintegrin echistatin, which preferentially inhibits the binding of ligands to $\beta 1$ - or $\beta 3$-containing integrins, was inefficient at blocking maturation rules out the involvement of $\alpha 3 \beta 1$ in mediating the effect of Reelin during maturation. Several lines of evidence indicate that the Src family kinases are involved in the Reelin transduction pathway. For instance, activation of the Src family kinases by Reelin is dependent on the receptors ApoER2 and VLDLR (Bock and Herz, 2003). Thus our data showing that inhibition of the Src kinase pathway and blockade of the receptors ApoER2 and VLDLR prevented the maturation-induced changes in NMDAR subunit composition are in agreement with a role of Reelin in this phenomenon.

Our data show that the low ifenprodil sensitivity of 9-12 DIV neurons was not perturbed by chronic blockade of either neuro- 
nal activity or activity of NMDARs and non-NMDARs, suggesting that neither neuronal activity nor activation of ionotropic glutamate receptors is necessary for the maintenance of NMDAR subunit composition between 9 and 12 DIV. These findings are consistent with the fact that the mechanisms underlying the release of Reelin are independent of depolarization, AMPA receptor activity, or NMDAR activity and, instead, involve a constitutive pathway (Lacor et al., 2000).

Reelin is a secreted protein (D'Arcangelo et al., 1997) that mediates its physiological functions on target cells via the extracellular milieu. We hypothesize that in our cultures the population of neurons showing an intense Reelin staining in the somatic compartment is the source of secreted Reelin. We also showed that the content of Reelin in the medium of cultured hippocampal neurons increased during maturation. We speculate that the discrete population of Reelin-immunopositive neurons controls the maturation-induced changes in NMDAR subunits of the neighboring neuronal network. Consistent with this hypothesis, we found that most (if not all) NR1-containing neurons also express the Reelin receptors ApoER2 or VLDLR. This expression pattern corroborates our electrophysiological data showing that after chronic treatments, the ifenprodil sensitivity of NMDA responses was perturbed in almost all of the neurons that were recorded. Thus we propose a model in which Reelin secreted by a discrete population of cells binds to a vast majority of neurons expressing VLDLR/ApoER2 and controls their somatic NMDAR subunit composition.

The same signaling pathway has been shown to play a role in neuronal migration (Drakew et al., 2002), neuritic outgrowth (Niu et al., 2004), and LTP (Weeber et al., 2002) in the hippocampus. Here we reveal a novel activity of Reelin and a new mode of control of NMDAR subunit composition. The present demonstration of the role of Reelin in postnatal maturation of hippocampal neurons reinforces the idea that ECM proteins in general and Reelin in particular are fundamental to neuronal functions throughout the entire lifespan of neuronal networks.

\section{References}

Alcantara S, Ruiz M, D’Arcangelo G, Ezan F, de Lecea L, Curran T, Sotelo C, Soriano E (1998) Regional and cellular patterns of reelin mRNA expression in the forebrain of the developing and adult mouse. J Neurosci 18:7779-7799.

Ali DW, Salter MW (2001) NMDA receptor regulation by Src kinase signalling in excitatory synaptic transmission and plasticity. Curr Opin Neurobiol 11:336-342.

Arnaud L, Ballif BA, Forster E, Cooper JA (2003) Fyn tyrosine kinase is a critical regulator of Disabled-1 during brain development. Curr Biol 13:9-17.

Audinat E, Lambolez B, Rossier J, Crepel F (1994) Activity-dependent regulation of $N$-methyl-D-aspartate receptor subunit expression in rat cerebellar granule cells. Eur J Neurosci 6:1792-1800.

Barria A, Malinow R (2002) Subunit-specific NMDA receptor trafficking to synapses. Neuron 35:345-353.

Beffert U, Stolt PC, Herz J (2004) Functions of lipoprotein receptors in neurons. J Lipid Res 45:403-409.

Biederer T, Sara Y, Mozhayeva M, Atasoy D, Liu X, Kavalali ET, Sudhof TC (2002) SynCAM, a synaptic adhesion molecule that drives synapse assembly. Science 297:1525-1531.

Bock HH, Herz J (2003) Reelin activates SRC family tyrosine kinases in neurons. Curr Biol 13:18-26.

Chavis P, Westbrook G (2001) Integrins mediate functional pre- and postsynaptic maturation at a hippocampal synapse. Nature 411:317-321.

Chun D, Gall CM, Bi X, Lynch G (2001) Evidence that integrins contribute to multiple stages in the consolidation of long-term potentiation in rat hippocampus. Neuroscience 105:815-829.

D’Arcangelo G, Curran T (1998) Reeler: new tales on an old mutant mouse. BioEssays 20:235-244.
D’Arcangelo G, Nakajima K, Miyata T, Ogawa M, Mikoshiba K, Curran T (1997) Reelin is a secreted glycoprotein recognized by the CR-50 monoclonal antibody. J Neurosci 17:23-31.

D’Arcangelo G, Homayouni R, Keshvara L, Rice DS, Sheldon M, Curran T (1999) Reelin is a ligand for lipoprotein receptors. Neuron 24:471-479.

Dityatev A, Schachner M (2003) Extracellular matrix molecules and synaptic plasticity. Nat Rev Neurosci 4:456-468.

Drakew A, Deller T, Heimrich B, Gebhardt C, Del Turco D, Tielsch A, Forster E, Herz J, Frotscher M (2002) Dentate granule cells in reeler mutants and VLDLR and ApoER2 knockout mice. Exp Neurol 176:12-24.

Dulabon L, Olson EC, Taglienti MG, Eisenhuth S, McGrath B, Walsh CA, Kreidberg JA, Anton ES (2000) Reelin binds $\alpha 3 \beta 1$ integrin and inhibits neuronal migration. Neuron 27:33-44.

Hanke JH, Gardner JP, Dow RL, Changelian PS, Brissette WH, Weringer EJ, Pollok BA, Connelly PA (1996) Discovery of a novel, potent, and Src family-selective tyrosine kinase inhibitor. Study of Lck- and FynTdependent T cell activation. J Biol Chem 271:695-701.

Herz J, Goldstein JL, Strickland DK, Ho YK, Brown MS (1991) 39-kDa protein modulates binding of ligands to low density lipoprotein receptorrelated protein/alpha 2-macroglobulin receptor. J Biol Chem 266:21232-21238.

Heynen AJ, Quinlan EM, Bae DC, Bear MF (2000) Bidirectional, activitydependent regulation of glutamate receptors in the adult hippocampus in vivo. Neuron 28:527-536.

Hiesberger T, Trommsdorff M, Howell BW, Goffinet A, Mumby MC, Cooper JA, Herz J (1999) Direct binding of Reelin to VLDL receptor and ApoE receptor 2 induces tyrosine phosphorylation of Disabled-1 and modulates tau phosphorylation. Neuron 24:481-489.

Hoffmann H, Gremme T, Hatt H, Gottmann K (2000) Synaptic activitydependent developmental regulation of NMDA receptor subunit expression in cultured neocortical neurons. J Neurochem 75:1590-1599.

Jossin Y, Ogawa M, Metin C, Tissir F, Goffinet AM (2003) Inhibition of SRC family kinases and nonclassical protein kinases $\mathrm{C}$ induce a reeler-like malformation of cortical plate development. J Neurosci 23:9953-9959.

Jossin Y, Ignatova N, Hiesberger T, Herz J, Lambert de Rouvroit C, Goffinet AM (2004) The central fragment of Reelin, generated by proteolytic processing in vivo, is critical to its function during cortical plate development. J Neurosci 24:514-521.

Lacor PN, Grayson DR, Auta J, Sugaya I, Costa E, Guidotti A (2000) Reelin secretion from glutamatergic neurons in culture is independent from neurotransmitter regulation. Proc Natl Acad Sci USA 97:3556-3561.

Lambert de Rouvroit C, de Bergeyck V, Cortvrindt C, Bar I, Eeckhout Y, Goffinet AM (1999) Reelin, the extracellular matrix protein deficient in reeler mutant mice, is processed by a metalloproteinase. Exp Neurol 156:214-217.

Legendre P, Rosenmund C, Westbrook GL (1993) Inactivation of NMDA channels in cultured hippocampal neurons by intracellular calcium. J Neurosci 13:674-684.

Miyata T, Nakajima K, Mikoshiba K, Ogawa M (1997) Regulation of Purkinje cell alignment by Reelin as revealed with CR-50 antibody. J Neurosci 17:3599-3609.

Monyer H, Burnashev N, Laurie DJ, Sakmann B, Seeburg PH (1994) Developmental and regional expression in the rat brain and functional properties of four NMDA receptors. Neuron 12:529-540.

Nakajima K, Mikoshiba K, Miyata T, Kudo C, Ogawa M (1997) Disruption of hippocampal development in vivo by CR-50 mAb against Reelin. Proc Natl Acad Sci USA 94:8196-8201.

Niu S, Renfro A, Quattrocchi CC, Sheldon M, D’Arcangelo G (2004) Reelin promotes hippocampal dendrite development through the VLDLR/ ApoER2-Dab1 pathway. Neuron 41:71-84.

Quinlan EM, Lebel D, Brosh I, Barkai E (2004) A molecular mechanism for stabilization of learning-induced synaptic modifications. Neuron 41:185-192.

Scotti AL, Herrmann G (2002) Reelin immunoreactivity in dissociated cultures of the postnatal hippocampus. Brain Res 924:209-218.

Senzaki K, Ogawa M, Yagi T (1999) Proteins of the CNR family are multiple receptors for Reelin. Cell 99:635-647.

Thibault G (2000) Sodium dodecyl sulfate-stable complexes of echistatin and RGD-dependent integrins: a novel approach to study integrins. Mol Pharmacol 58:1137-1145. 
Triller A, Choquet D (2005) Surface trafficking of receptors between synaptic and extrasynaptic membranes: and yet they do move! Trends Neurosci 28:133-139.

Trommsdorff M, Gotthardt M, Hiesberger T, Shelton J, Stockinger W, Nimpf J, Hammer RE, Richardson JA, Herz J (1999) Reeler/Disabled-like disruption of neuronal migration in knockout mice lacking the VLDL receptor and ApoE receptor 2. Cell 97:689-701.

Vicini S, Wang JF, Li JH, Zhu WJ, Wang YH, Luo JH, Wolfe BB, Grayson DR (1998) Functional and pharmacological differences between recombinant $N$-methyl-D-aspartate receptors. J Neurophysiol 79:555-566.

Weeber EJ, Beffert U, Jones C, Christian JM, Forster E, Sweatt JD, Herz J (2002) Reelin and ApoE receptors cooperate to enhance hippocampal synaptic plasticity and learning. J Biol Chem 277:39944-39952.

Wenthold RJ, Prybylowski K, Standley S, Sans N, Petralia RS (2003)
Trafficking of NMDA receptors. Annu Rev Pharmacol Toxicol 43:335-358.

Wenzel A, Fritschy JM, Mohler H, Benke D (1997) NMDA receptor heterogeneity during postnatal development of the rat brain: differential expression of the NR2A, NR2B, and NR2C subunit proteins. J Neurochem 68:469-478.

Williams K (1993) Ifenprodil discriminates subtypes of the $N$-methyl-Daspartate receptor: selectivity and mechanisms at recombinant heteromeric receptors. Mol Pharmacol 44:851-859.

Williams K, Russell SL, Shen YM, Molinoff PB (1993) Developmental switch in the expression of NMDA receptors occurs in vivo and in vitro. Neuron 10:267-278.

Yu XM, Askalan R, Keil II GJ, Salter MW (1997) NMDA channel regulation by channel-associated protein tyrosine kinase Src. Science 275:674-678. 